\title{
Comparative Study between Various Topological Models of Base Stations in Cellular Mobile Radio Communication
}

\author{
Mohammed Fawzi Alhunaity \\ Faculty of Science and Information Technology, Al-Balqa’ Applied University, Al-Salt 19117, Jordan
}

\begin{abstract}
In this paper, we analyze the different parameters of quality for the network models of base stations (BS) when the service messages have not a delay. Also, we compare between four topologies star, ring, radial ring and fully connected using identical quality of user service and conditional cost of one Erlang. Our results have been obtained for different probabilities of failure in service message on one branch and fixed number of web channels. The obtained results show that radial ring topology outperform better than the others with respect to both cost and hierarchy.
\end{abstract}

Key words: Cellular mobile communication, topology, base station, probability of failure

\section{INTRODUCTION}

The traditional problem faced by mobile radio system (MRS) designers is concerned with how to balance the apparently conflicting requirements of area coverage and user capacity. These requirements conflict arise because if $\mathrm{BS}$ is to provide service to mobiles over a wide area, it must have high power and be situated on the highest point available in the required coverage area. But following this strategy means that the channels allocated to the transmitting site cannot be reused for another service for a very considerable distance. Since the spectrum available is fixed and the traditional technologies using amplitude and frequency modulations have dictated division of the spectrum into channel segments on a frequency division-multiplexing basis, only a small total user population per channel can be achieved by the high-power hilltop site approach. Recognizing this, the regulatory authorities have, since the early introduction of MRS on a substantial scale, restricted BS transmitting power in order to improve frequency re-use opportunities and this has obliged system designers to follow other strategies to achieve area coverage. These involve developing an infrastructure of fixed radio or line links to connect a number of BS via a central control point, so that the service transmissions can be radiated simultaneously between BS to locate a wanted mobile. Then, if appropriate, a selection procedure can be utilized to use only the nearest base to the mobile to sustain subsequent message exchanges. Clearly, creating national networks by this technique is costly and complicated and, in any case, an obvious alternative is available. A national network of immense capacity already exists in the public switched telephone network (PSTN). This could be used, as the basic infrastructure to connect BS together if there are many connection points between BS and fixed network, then individual
BS need cover only small areas and enormously greater frequency re-use becomes possible ${ }^{[1]}$.

Analysis of the network models of BS: In the correspondence with the main concepts and principles of construction of cellular MRS, the BS of macro zone is linked among themselves through the PSTN, or with the help of separate radio channels. The web with hexagonal cells and three levels of BS hierarchy are considered in work ${ }^{[2,3]}$. There are various hierarchal in which BS are distributed such as: BS of macro zone, central BS (CBS), main BS of a radio network. Moreover the topological web structure models of BS that represents practical interest and presented $\mathrm{in}^{[4]}$ are shown in Fig. 1(a-d).

For the web structures shown in Fig. 1 when the service messages are assumed to be without delay, we use the following parameters of quality in our analysis:

* $\mathrm{Pv}$ - probability of failure in service message on one branch;

* PvT- probability of failure in connection between BS zones;

* Pvy- probability of failure in connection between $\mathrm{BS}$ and CBS;

* Mo- quantity of branches in web structure;

* MT- the average of intermediate branches, on which passes the message between the users of macro zone;

* My- the average of branches, by which passes the messages to connect BS with CBS;

* YB- general average load on branch;

* YT- the average load on branch in one direction created transit through CBS by the messages of macro zone;

* Yy- the average load on branch in one direction created by the messages between BS and CBS;

* SM- cost of one-minute occupation of the channel; 


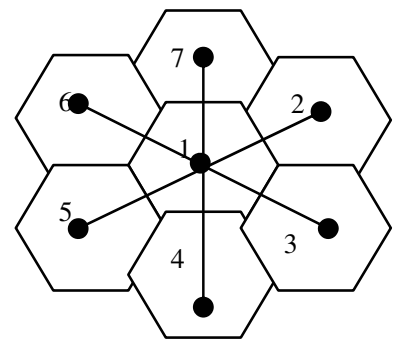

(a)

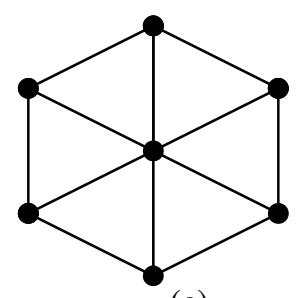

(c)

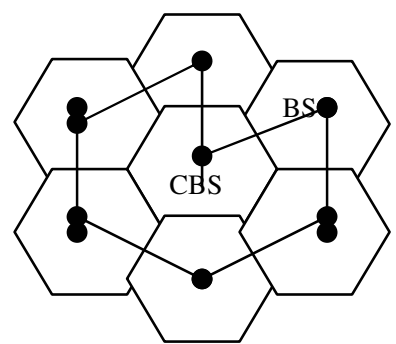

(b)

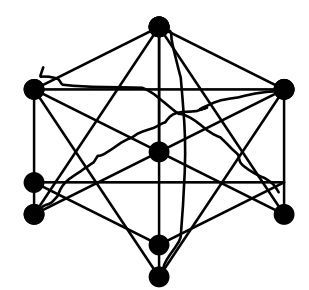

(d)

Fig. 1: Network models of BS in cellular MRS (a) Star (b) Ring (c) Radial ring (d) Fully connected

* VTP- number of web structure channels demanded for provision of a specific level of losses in required service quality $(\mathrm{Pv})$

* L-general length of web channels.

When we use the techniques presented in $^{[5]}$ to calculate the above assumed parameters under the condition of service quality and number of $\mathrm{BS}(\mathrm{Pv}<<1$ and $\mathrm{N}=7$ ), we get the characteristics of topological web structure models of BS as shown in Table 1.

From Table 1, it is shown that:

The Star configuration of a web has an obvious advantage on efficiency of communication with a central knot and least vitality among all structures, which were indicated on Fig. 1. The greatest vitality and reliability in delivery of information was obtained from a fully connected model. In this model, the principle of "each with each" will be realized and the total number of communication lies between BS makes $\mathrm{M}=\mathrm{N}(\mathrm{N}-1) / 2=7(6) / 2=21$, that is 3 times more, than ring and in 3.5 times more, than star. For fully connected model, the probability of failure in connection between BS was determined by Bernoulli distributions for (N-2) of parallel circuitous paths from two branches ${ }^{[6]}$.

* For a radial ring model there are typical advantages of ring, star and partial of fully connected webs. It is stipulated by that the topology radial ring web on one hand is superposition of ring and star, but on the other hand is planar polygonal part of a fully connected model. For a radial ring web the magnitude PvT is determined provided that in case of engaged direct path is selected at first, two links of a circuitous path and then (in case of engaged two links) three links.
* In the case of equal significance's $\mathrm{Pv}$ and $\mathrm{Y}$ for both ring and star topologies, the cost of these two topologies is adequate. Also, in the ring topology, the possibility of passing message in two opposite directions let the load on a communication branch is less by $150 \%$ than the star. Finally, the probability of failure in communication in the ring is almost less than the star.

* The load on a branch in fully connected model is almost three times less, than in radial ring. However, it is achieved at the expense of large number of circuitous channels and magnification of cost of network. Thus, if BS of macro zone is attached to develop PSTN, it is expedient to use not all branches of the fully connected model, but only those make a structure close to radial ring. Moreover, for the fully connected topology, analytical result indicate that when the load across the branch lies between 0.17 to 0.6 , the cost of a web (fully connected) will decreases to 1.7 times (21/12 times), the other two topologies and the quality of services is worsened.

Conditions of comparative analysis between the various models: Using an Erlang (the unit of traffic) definition where the average proportion of time for which the branch is busy ${ }^{[7]}$, a comparison of star and ring models on a criterion of general length of all web channels $\mathrm{W}=\mathrm{Lstar} /$ Lring under number of BS Nstar $=$ Nring, $\mathrm{Pv}=10^{-2}$ and identical loads in Erlang $(\mathrm{Y})$ on a knot show that (Fig. 2$)^{[8]}$.

If $\mathrm{N} \geq 5$ the star web concedes ring and the more considerably than it is, more $\mathrm{N}$ and less $\mathrm{Y}$. 
Table 1: Characteristic of the topological network models of BS

\begin{tabular}{|c|c|c|c|c|}
\hline Characteristic & Type of moc & & & \\
\hline & Star & Ring & Radial ring & Fully connected \\
\hline Mo & $\mathrm{N}-1=6$ & $\mathrm{~N}=7$ & $2(\mathrm{~N}-1)=12$ & $\frac{N(N-1)}{2}=21$ \\
\hline MT 2(1- Pv) & $\frac{N+1}{4}=2$ & $(1-P v)(1+P v)^{2}$ & $1+\mathrm{Pv}$ & \\
\hline My & $1-\mathrm{Pv}$ & $\frac{N+1}{4}=2$ & $(1-\mathrm{Pv})\left(1+2 \mathrm{Pv}+7 \mathrm{Pv}^{3}\right)$ & $1+\mathrm{Pv}$ \\
\hline $\mathrm{PvT}$ & Pv (2- Pv) & $\frac{N(N+1)}{N-1} \operatorname{Pv}^{2}$ & $9 \mathrm{Pv}^{3}(2-\mathrm{Pv})$ & $\mathrm{Pv}^{N-1}(2-\mathrm{Pv})^{N-2}$ \\
\hline Pvy & $\mathrm{Pv}$ & $\frac{N^{2}}{4} \mathrm{PV}^{2}$ & $\mathrm{Pv}^{2}(1+2 \mathrm{Pv})$ & $\mathrm{Pv}^{N-1}(2-\mathrm{Pv})^{N-2}$ \\
\hline YT & YT (1- Pv) & $\frac{Y}{1-P_{V}}$ & $\frac{N \cdot Y\left(1+P_{V}^{2}\right)}{4(N-1)}$ & $\frac{Y}{N-1}(1+2 \mathrm{Pv})$ \\
\hline Yy & Yy/2 & $\frac{Y}{1-P_{V}}$ & $\frac{N \cdot Y\left(1+2 P_{V}+7 P_{V}{ }^{3}\right)}{4(N-1)}$ & $\frac{Y}{N-1}(1+2 \mathrm{Pv})$ \\
\hline YB & $Y(1.5-\mathrm{Pv})$ & $\frac{Y}{1-P_{V}}$ & $\frac{N \cdot Y}{2(N-1)}(1+2 \mathrm{Pv})$ & $\frac{Y}{N-1}(1+2 \mathrm{Pv})$ \\
\hline$\underline{\text { SM }}$ & $\mathrm{S}(\mathrm{N}-1)=6 \mathrm{~S}$ & $\mathrm{SN}=7 \mathrm{~S}$ & $\mathrm{~S} \cdot 2(\mathrm{~N}-1)=12 \mathrm{~S}$ & $\mathrm{SN}(\mathrm{N}-1) / 2=21 \mathrm{~S}$ \\
\hline
\end{tabular}

Table 2: Characteristics of topological models at $\mathrm{Pv}=10^{-2}$ and $\mathrm{N}=7$

\begin{tabular}{lccc}
\hline Type of model & Conditional cost, $\mathrm{S}$ & Average value of probability of failure in communication, $\mathrm{Pv}$ & Average load on branch, Y \\
\hline Star & $6 \mathrm{~S}$ & $\operatorname{Pv}(1-2 \mathrm{Pv})=9.8 \cdot 10^{-3}$ & $1.49 \mathrm{Y}$ \\
Ring & $7 \mathrm{~S}$ & $\frac{N(N+1)}{N-1} \mathrm{Pv}^{2}=9.33 \cdot 10^{-4}$ & $1.01 \mathrm{Y}$ \\
Radial ring & $12 \mathrm{~S}$ & $\mathrm{Pv}^{2}(1+2 \mathrm{Pv})=1.02 \cdot 10^{-4}$ & $0.6 \mathrm{Y}$ \\
Fully connected & $21 \mathrm{~S}$ & $\mathrm{Pv}^{N-1}(2-\mathrm{Pv})$ & \\
\end{tabular}

Table 3: Characteristics of topological models at $\mathrm{Pv}=10^{-2}$ and $\mathrm{Pv}=10^{-3}$ for $\mathrm{V}=12$

\begin{tabular}{lcccccc}
\hline Type of model & Load $\mathrm{Y}$ at $\mathrm{Pv}=10^{-2}$ & Load on Web & The price of one Erlang & Load $\mathrm{Y}$ at $\mathrm{Pv}=10^{-3}$ & Load on web & The price of one Erlang \\
\hline Star & 8.67 & 52.02 & $0.115 \mathrm{~S}$ & 6.35 & 38.10 & $0.157 \mathrm{~S}$ \\
Ring & 5.88 & 40.75 & $0.172 \mathrm{~S}$ & 4.23 & 29.58 & $0.237 \mathrm{~S}$ \\
Radial ring & 3.49 & 41.88 & $0.287 \mathrm{~S}$ & 2.54 & 30.48 & $0.394 \mathrm{~S}$ \\
Fully connected & 0.99 & 20.79 & $1.01 \mathrm{~S}$ & 0.72 & 15.12 & $1.388 \mathrm{~S}$ \\
\hline
\end{tabular}

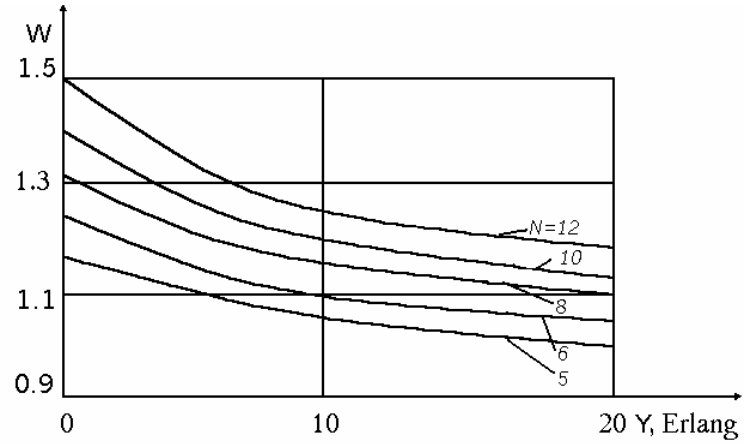

Fig. 2: Relation between $\mathrm{Y}$ and $\mathrm{W}$ in star and ring models for various values of $\mathrm{N}$

If $\mathrm{N}>15$ and $\mathrm{Y}>1.5$ Erlang, the magnitude of a penalty makes less than $25 \%$.

If $\mathrm{N}=7$ and $\mathrm{Y}=5$ to 20 Erlang, the magnitude of a penalty lies within the limits of 9 to $15 \%$.
We compare now models in which the following condition is satisfied:

$\mathrm{Pv}($ star $)=\mathrm{Pv}($ ring $)=\mathrm{Pv}($ radial ring $)=\mathrm{Pv}($ fully connected $)$

With this purpose, we shall define average permissible loads on one knot. Let's accept for a definiteness amount of channels in a branch $\mathrm{V}=12$ and $\mathrm{Pv}=10^{-2}$. For ensuring of such degree of service on a ring web, it is necessary to have an information load of the first kind Yring $\leq 5.88$ Erlang. The load on a knot will make $\mathrm{Yy}=$ Yring $(1-\mathrm{Pv})=5.88(0.99)=5.82$ Erlang. A general load on a web $\mathrm{Y}=7 \mathrm{Yy}=7(5.82)=$ 40.75 Erlang. Conditional costs of this load 7S unit. Conditional cost of one Erlang $7 \mathrm{~S} / \mathrm{Y}=7 \mathrm{~S} / 40.75=$ $0.172 \mathrm{~S}$. In a star model, the load on the branch is great, unlike the radial ring and fully connected models in which the load is little. 


\section{RESULTS}

Comparing the different topological network models of BS under condition of different $\mathrm{Pv}$ with the conditional cost of one Erlang and load on web structures, we have the following results of recalculations for characteristics of models at $\mathrm{Pv}=10^{-2}$ and $\mathrm{Pv}=10^{-3}$ for $\mathrm{V}=12$ executed on the formulas of Table 2 (Table 3).

Analyses of our obtained results permit us making practical important conclusions giving by the following:

* The comparison between topological models of BS when $\mathrm{Pv}=$ const shows, that the least price of transfer one Erlang is in star model and the greatest is in fully connected one and the difference almost of one order.

* The comparison between topological models of BS when $\mathrm{Pv} \neq$ const essentially Changes the results, the most effective from compared models will be radial ring, the Price of one Erlang becomes commensurable.

\section{REFERENCES}

1. Alberto, L.-G. and I. Widjaja, 2000. Communication Networks. Singapore, McGrawHill.

2. Ramsdale, P.A., 1996. The development of personal communications. Electron. Commun. Eng. J., IEE., pp:143-151.

3. Padgett, J.E., C.G. Guenther and T. Hattori, 1995. Overview of wireless personal communications. IEEE Commun. Mag., 33: 28-41.

4. Behrouz, A.F., 2001. Data Communications and Networking. Singapore, McGraw-Hill.

5. William, C.Y.L., 1997. Mobile Communications Engineering. New York, McGraw-Hill.

6. William, S., 1997. Data and Computer Communications. USA, Prentice Hall.

7. Flood, J.E., 199. Telecommunications Switching, Traffic and Networks. Manchester, Prentice Hall.

8. Cho, Y.J., Y.B. Lin and C.H. Rao, 1997. Reducing the Network Cost of Call Delivery to GSM Roamers. IEEE Network, 11: 19-25. 